\title{
EXACT EDGE WAVE SOLUTIONS FOR SOME GENERALISED EXPONENTIAL SHELF TOPOGRAPHIES
}

\author{
J. P. LOUIS ${ }^{1}$ AND D. J. CLARKE ${ }^{2}$
}

(Received 23 November 1984; revised 18 April 1985)

\begin{abstract}
Exact wave-height solutions are presented for trapped waves over two new three-parameter depth topographes. Dispersive properties are calculated for both a semi-infinite and a truncated convex exponential profile, as well as for a semi-infinite concave profile. The analysis in all three cases is general in that both horizontal divergence and rotational effects are included. These solutions may be used for either high-frequency edge wave or low-frequency shelf wave studies by taking appropriate limits $(f \rightarrow 0$ for edge wave and $\varepsilon=f^{2} L^{2} / g H \ll 1$ for shelf waves).
\end{abstract}

\section{Introduction}

The reflection of high-frequency gravity waves at a coastal boundary, coupled with refraction effects due to shelving bottom topography, gives rise to a class of coastally trapped waves known as edge waves. A similar trapping situation also occurs when low-frequency topographic Rossby waves are reflected from a lateral boundary. In the latter case the resulting trapped waves are traditionally referred to as shelf waves.

A number of exact edge wave solutions have been derived for a variety of simple bottom topographies. Two of the most useful models have been the convex exponential shelf topography

$$
h(x)=h_{0} e^{a x}
$$

(see for example, [2], [4] and [7]) and the concave exponential beach topography

$$
h(x)=h_{0}\left(1-e^{-a x}\right)
$$

\footnotetext{
${ }^{1}$ School of Applied Science Riverina-Murray Institute of Higher Education. P. O. Box 588, Wagga Wagga, N. S. W. 2650.

${ }^{2}$ Department of Mathematics, The University of Wollongong, Wollongong, N. S. W. 2500.

(C) Copyright Australian Mathematical Society 1986, Serial-fee code 0334-2700/86
} 
(see for example, [1] and [6]). One disadvantage with these model topographies is that the two adjustable parameters $h_{0}$ and $a$ do not allow for independent specification of the three physical shelf parameters initial depth at the coastline $h_{s}$, shelf slope $h_{s}^{\prime}$, and shelf width $L$ (or alternatively the depth at the edge of the shelf, $h_{L}$ ).

In this paper the dispersion relationships are presented for a generalized (three-parameter) convex exponential shelf profile,

$$
h(x)=h_{0}\left(\alpha e^{a x}-1\right)
$$

as well as for a generalized concave exponential beach profile,

$$
h(x)=h_{0}\left(1-\beta e^{-a x}\right) .
$$

For both profiles, the analysis is general in the sense that it includes the horizontal divergence term in the continuity equation as well as the Coriolis terms in the horizontal momentum equations. In practice, horizontal divergence can be neglected for low-frequency shelf waves, while rotational effects can be neglected in the high-frequency edge wave limit.

\section{Differential equation and boundary conditions}

Consider the coastal geometry shown in Figure 1, in which a homogeneous ocean is bounded by a straight coastline whose bottom topography varies in the offshore direction only.

Neglecting vertical accelerations ( $w \ll u, v)$ and assuming that displacements in the free surface elevation are small compared to the depth of the water column $(\zeta \ll h)$, then the horizontal momentum equations for this system may be

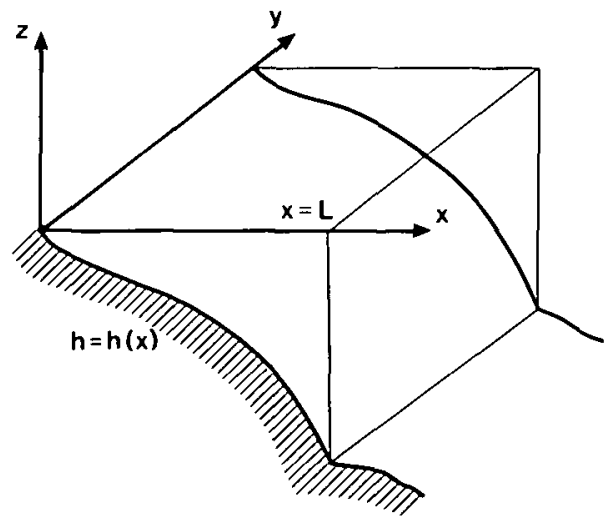

Figure 1. Shelf geometry and axis system orientation. 
approximated by

$$
\begin{aligned}
& u_{t}-f v=-g \zeta_{x}, \\
& v_{t}+f u=-g \zeta_{y},
\end{aligned}
$$

where $f$ is the Coriolis parameter and $g$ the acceleration due to gravity. To the same level of approximation the vertically integrated continuity equation may be written as,

$$
(h u)_{x}+(h v)_{y}=-\zeta_{t} .
$$

Periodic edge-wave solutions in this system are found by letting

$$
u, v, \zeta \propto \exp [i(m y-\sigma t)],
$$

where $m$ is assumed to be positive always, so that $\sigma<0$ represents a wave travelling in the negative $y$ direction. Combining (5), (6) and (7) yields the differential equation,

$$
\frac{d}{d x}\left[h \frac{d \zeta}{d x}\right]+\left[\left(\sigma^{2}-f^{2}\right) / g-m^{2} h-\frac{m f}{\sigma} \frac{d h}{d x}\right] \zeta=0,
$$

which, together with the appropriate boundary conditions, governs the fluid motion over the margin.

Physically acceptable solutions of (8) must require that $\zeta$ is finite at all times. Also, at a rigid wall it is required that the normal component of mass flow is zero. Hence the boundary conditions at the coast are taken as,

$$
\frac{d \zeta}{d x}-\frac{m f}{\sigma} \zeta=0, \text { at } x=0,
$$

where the bottom depth is non-zero at the coast, or as

$$
\zeta \text { finite, at } x=0,
$$

where the bottom depth is zero at the coastal boundary. In the case of shelf waves, the requirement that no waves propagate past the shelf is equivalent to (9).

For a semi-infinite continental margin the boundary condition for coastally trapped waves requires that the horizontal transport, $|h \mathbf{u}| \rightarrow 0$ as $x \rightarrow \infty$. From (5) and (7) this requires that,

$$
h \zeta, h \frac{d \zeta}{d x} \rightarrow 0, \text { as } x \rightarrow \infty .
$$

However, when the continental shelf is not semi-infinite in extent, but is truncated at $x=L$ by an ocean of constant depth $(H=h(L))$, the requirement for continuity of wave height and the normal component of volume flux across the boundary leads to the alternative offshore boundary condition,

$$
\frac{d \zeta}{d x}+r \zeta=0, \text { at } x=L
$$


where

$$
r^{2}=\left(m^{2}+\left(f^{2}-\sigma^{2}\right) / g H\right)>0
$$

\section{Convex exponential shelf profile}

Consider the general, three-parameter, convex exponential shelf profile given by equation (3). For this profile the physical shelf parameters $h_{s}, h_{s}^{\prime}$ and $h_{L}$ are related to the model shelf parameters, $h_{0}, \alpha$ and $a$ by

$$
\begin{aligned}
& h_{s}=h_{0}(\alpha-1), \\
& h_{s}^{\prime}=h_{0} \alpha a \text { and } h_{L}=h_{0}\left(\alpha e^{a L}-1\right) .
\end{aligned}
$$

Figure 2 displays the values of the quantities $h_{0}, \alpha$ and $a$ for independent perturbations of the physical shelf parameters away from those used by [2], $h_{s}=70 \mathrm{~m}, h_{s}^{\prime}=3.735 \times 10^{-3}$ and $h_{L}=5 \mathrm{~km}$ where $L=80 \mathrm{~km}$, to approximate the continental margin near Sydney. The two parameter exponential profile (1) may be considered as a limiting form of the profile (3) when $\alpha \rightarrow \infty, h_{0} \rightarrow 0$ while the product $h_{0} \alpha$ remains constant. Dispersion relationships will be derived

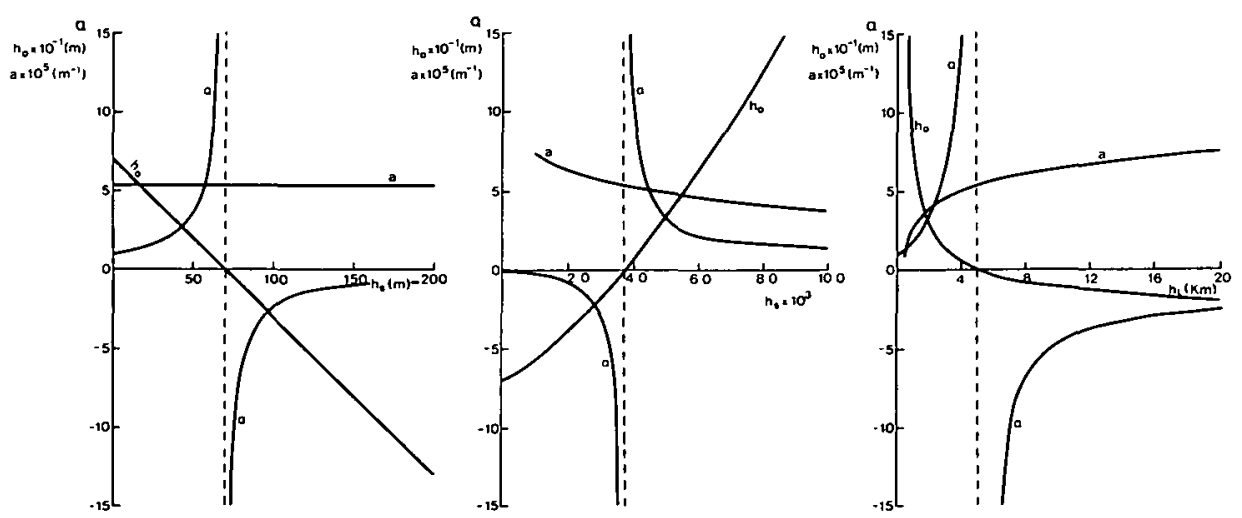

Figure 2. Behavior of the convex exponential shelf parameters $h_{0}, \alpha$ and $a$ for independent variation in:
(a) $h_{s}$ with $h_{s}^{\prime}=3.735 \times 10^{-3}$ and $h_{L}=5 \mathrm{~km}$.
(b) $h_{s}^{\prime}$ with $h_{s}=70 \mathrm{~m}$ and $h_{L}=5 \mathrm{~km}$.
(c) $h_{L}$ with $h_{s}=70 \mathrm{~m}$ and $h_{s}^{\prime}=3.735 \times 10^{-3}$. 
for both the semi-infinite shelf

$$
h=h_{0}\left(\alpha e^{a x}-1\right), \quad 0 \leqslant x<\infty,
$$

as well as for the truncated exponential profile

$$
h= \begin{cases}h_{0}\left(\alpha e^{a x}-1\right), & 0 \leqslant x<L, \\ h_{L}, & x \geqslant L .\end{cases}
$$

Substitution of (3) into (8) yields the differential equation governing the motion of edge waves over the shelf region as

$$
\begin{aligned}
\left(\alpha e^{\alpha x}-1\right) & d^{2} \zeta / d x^{2}+\alpha a e^{a x} d \zeta / d x \\
& +\left\{\left(\sigma^{2}-f^{2}\right) /\left(g h_{0}\right)-m^{2}\left(\alpha e^{a x}-1\right)-\frac{m f}{\sigma} a \alpha e^{a x}\right\} \zeta=0 .
\end{aligned}
$$

The change of variables

$$
X=e^{-a x} / \alpha,
$$

and

$$
Z=X^{-p} \zeta
$$

where

$$
p=(1+\nu) / 2
$$

with

$$
\nu^{2}=4 m^{2} / a^{2}+4 m f /(a \sigma)+1,
$$

transforms (17) into the hypergeometric differential equation

$$
X(1-X) d^{2} Z / d X^{2}+[2 p-(2 p+1) X] d Z / d X-\left(p^{2}-q^{2}\right) Z=0,
$$

where

$$
q^{2}=\left(\sigma^{2}-f^{2}\right) /\left(a^{2} g h_{0}\right)+m^{2} / a^{2} .
$$

Similarly, the semi-infinite exponential shelf has the transformed depth profile

$$
h=h_{0}\left(X^{-1}-1\right), \quad 0<X \leqslant \alpha^{-1},
$$

while the truncated one has the profile

$$
h=\left\{\begin{array}{l}
h_{0}\left(X^{-1}-1\right), \quad \Delta<X \leqslant \alpha^{-1}, \\
h_{L}, \quad X \leqslant \Delta,
\end{array}\right.
$$

where

$$
\Delta=e^{-a L} / \alpha .
$$

One solution of (22) may now be written in terms of the hypergeometric fucntion $F(a, b, c ; z)$ as

$$
Z=A F(p+q, p-q, 2 p ; X)
$$


When $a, b, c-a$ and $c-b$ are non-integers, two independent solutions of the hypergeometric differential equation are given by [5] as

$$
u_{1}(z)=F(a, b, c ; z) \text {, }
$$

and

$$
u_{2}(z)=z^{1-c} F(a-c+1, b-c+1,2-c ; z) .
$$

Thus, with (19) and (20), the general wave height solution over the shelf region (3) may be written as

$$
\begin{aligned}
\zeta= & A X^{(1+\nu) / 2} F\left(\frac{1}{2}+\frac{\nu}{2}+q, \frac{1}{2}+\frac{\nu}{2}-q, 1+\nu ; X\right) \\
& +B X^{(1+\nu) / 2} F\left(\frac{1}{2}-\frac{\nu}{2}+q, \frac{1}{2}-\frac{\nu}{2}-q, 1-\nu ; X\right),
\end{aligned}
$$

which, together with the appropriate boundary conditions, governs the dispersive properties of edge waves over the margin.

Since the depth profile (3) has a vertical barrier at $x=0$, the relevant boundary condition for trapped waves over the semi-infinite margin (15) are (9) and (11). For the truncated shelf, the off-shore trapping condition (11) is replaced by the boundary condition (12), at the edge of the shelf.

Transforming the boundary conditions (9), (11) and (12), by (18) yields the boundary conditions appropriate to the solution (28) as

$$
X \frac{d \zeta}{d X}+\frac{m f}{a \sigma} \zeta=0, \quad X=\alpha^{-1},
$$

at the coastline,

$$
X^{-1} \zeta, \frac{d \zeta}{d X} \rightarrow 0 \quad \text { as } X \rightarrow 0
$$

at infinity, and

$$
X \frac{d \zeta}{d X}-\frac{r}{a} \zeta=0, \quad X=\Delta,
$$

at the truncation $x=L$ and where $r^{2}$ is given by (13).

\section{Dispersion relation: semi-infinite convex shelf}

The general solution (28) satisfies the off-shore trapping condition (30), only when $B$ is zero and $\nu^{2}>1$. This gives the following bound, from (21),

$$
m / a+f / \sigma>0 \text {, }
$$

on frequency and wave number. This bound is the same as that obtained by [4] and eliminates the quasigeostrophic region of the $\sigma-m$ plane for edge waves over the semi-infinite exponential shelf $h=h_{0} e^{a x}$. 
Substitution of (28) with $B=0$ into the coastal boundary condition (29) yields the dispersion relation governing the discrete modes of oscillation over the semi-infinite margin (15) as

$$
\begin{aligned}
& {\left[p+\frac{m f}{a \sigma}\right] F\left(p+q, p-q, 2 p ; \alpha^{-1}\right)} \\
& \quad+\alpha^{-1}\left(p^{2}-q^{2}\right) /(2 p) F\left(p+q+1, p-q+1,2 p+1 ; \alpha^{-1}\right)=0,
\end{aligned}
$$

where the hypergeometric series in (33) are absolutely convergent for $|\alpha|>1$ and conditionally convergent for $|\alpha|=1$.

In the limit as $\alpha \rightarrow 1$, the shelf profile (15) reduces to the convex exponential shelf profile examined by [3], viz.

$$
h=h_{0}\left(e^{a x}-1\right), \quad 0 \leqslant x<\infty,
$$

having zero depth at the coastline. In this case the coastal boundary condition (29) is replaced by (10), i.e.,

$$
\zeta \text { finite at } X=1 \text {. }
$$

However, convergence of the solution (28) with $B=0$ at $X=1$ requires the hypergeometric series to terminate, i.e.,

$$
p-q=-n, \quad n=0,1,2, \ldots,
$$

which is the same dispersion relationship as that used by [3] to examine the properties of high frequency edge waves over the exponential shelf given by (34).

\section{Dispersion relation: truncated convex shelf}

Applying the boundary conditions (29) and (31) to the general wave height solution (28) yields

$$
\begin{aligned}
& A \alpha^{-p}\left\{\alpha^{-1} F_{1}^{\prime}\left(\alpha^{-1}\right)+\left(p+\frac{m f}{a \sigma}\right) F_{1}\left(\alpha^{-1}\right)\right\} \\
& +B \alpha^{p-1}\left\{\alpha^{-1} F_{2}^{\prime}\left(\alpha^{-1}\right)+\left(1-p+\frac{m f}{a \sigma}\right) F_{2}\left(\alpha^{-1}\right)\right\}=0
\end{aligned}
$$

and

where

$$
\begin{aligned}
A \Delta^{p}\left\{\Delta F_{1}^{\prime}(\Delta)+\left(p-\frac{r}{a}\right) F_{1}(\Delta)\right\} & \\
& +B \Delta^{1-p}\left\{\Delta F_{2}^{\prime}(\Delta)+\left(1-p-\frac{r}{a}\right) F_{2}(\Delta)\right\}=0
\end{aligned}
$$

$$
\begin{aligned}
& F_{1}(X)=F(p+q, p-q, 2 p ; X), \\
& F_{2}(X)=F(-p+q+1,-p-q+1,2-2 p ; X)
\end{aligned}
$$


and where a dash denotes differentiation with respect to $X$. The condition that equations (35) have a non-trivial solution yields the dispersion relation for edge waves over the truncated shelf profile (16) as

$$
\begin{aligned}
& \alpha^{-p} \Delta^{1-p}\left\{\alpha^{-1} F_{1}^{\prime}\left(\alpha^{-1}\right)+\left(p+\frac{m f}{a \sigma}\right) F_{1}\left(\alpha^{-1}\right)\right\} \\
& \left\{\Delta F_{2}^{\prime}(\Delta)+\left(1-p-\frac{r}{a}\right) F_{2}(\Delta)\right\}- \\
& \alpha^{p-1} \Delta^{p}\left\{\Delta F_{1}^{\prime}(\Delta)+\left(p-\frac{r}{a}\right) F_{1}(\Delta)\right\} \\
& \left\{\alpha^{-1} F_{2}^{\prime}\left(\alpha^{-1}\right)+\left(1-p+\frac{m f}{a \sigma}\right) F_{2}\left(\alpha^{-1}\right)\right\}=0 .
\end{aligned}
$$

\section{Concave exponential beach profile}

Consider a semi-infinite beach, whose depth profile in the off-shore direction is given by the generalized concave exponential form (4), i.e.

$$
h=h_{0}\left(1-\beta e^{-a x}\right), \quad 0 \leqslant x<\infty .
$$

For this profile, the parameters $h_{0}, a$ and $\beta$ are related to the physical parameters $h_{s}, h_{s}^{\prime}$ and $h_{\infty}$, used to specify the margin, by

$$
\begin{aligned}
h_{0} & =h_{\infty}, \\
a & =h_{s}^{\prime} /\left(h_{\infty}-h_{s}\right), \\
\beta & =1-h_{s} / h_{\infty},
\end{aligned}
$$

where $h_{\infty}$ is the limiting depth of the margin as $x \rightarrow \infty$. The two-parameter concave exponential depth profile considered by [1], viz.

$$
h=h_{0}\left(1-e^{-a x}\right), \quad 0 \leqslant x<\infty,
$$

may be considered as a limiting case of (37) as $\beta \rightarrow 1$ (i.e. $h_{s} \rightarrow 0$ ).

Substituting the exponential form (4) into (8) yields the differential equation for wave height as

$$
\begin{aligned}
& h_{0}\left(1-\beta e^{-a x}\right) d^{2} \zeta / d x^{2}+h_{0} \beta a e^{-a x} d \zeta / d x \\
& +\left[\left(\sigma^{2}-f^{2}\right) / g-m^{2} h_{0}\left(1-\beta e^{-a x}\right)-\frac{m f}{\sigma} h_{0} a \beta e^{-a x}\right] \zeta=0 .
\end{aligned}
$$

The transformations

$$
X=\beta e^{-a x},
$$

and

$$
Z=X^{-s \zeta}
$$


where

$$
s^{2}=m^{2} / a^{2}-\left(\sigma^{2}-f^{2}\right) / a^{2} g h_{0},
$$

reduce (40) into the hypergeometric differential equation

$$
\begin{aligned}
X(1-X) d^{2} Z / d X^{2}+[(2 s+1) & -(2 s+2) X] d Z / d X \\
& -\left(s^{2}-t^{2}+s+t\right) Z=0,
\end{aligned}
$$

where

$$
t=\left[1+\left(1-4\left(\frac{m f}{a \sigma}-m^{2} / a^{2}\right)\right)^{1 / 2}\right] / 2 .
$$

Thus, with (27) and (42), the general wave height solution, over the beach profile (37), may be written in terms of the hypergeometric function $F(a, b, c ; z)$ as

$$
\begin{aligned}
\zeta= & A X^{s} F(s+t, s-t+1,2 s+1 ; X) \\
& +B X^{-s} F(-s+t,-s-t+1,1-2 s ; X) .
\end{aligned}
$$

Using (41), the semi-infinite beach (37) has the transformed depth function

$$
h=h_{0}(1-X), \quad 0<X \leqslant \beta .
$$

Since this profile has a vertical barrier at the coastline $(X=\beta)$ the appropriate coastal boundary condition is (9). Also, since the depth function (47) tends to a finite depth at infinity $(X \rightarrow 0)$, the off-shore trapping condition (11) reduces to $\zeta \rightarrow 0$ as $x \rightarrow \infty$. Hence, using (41) again, the appropriate transformed boundary conditions for the semi-infinite beach (47) are

$$
X \frac{d \zeta}{d X}+\frac{m f}{a \sigma} \zeta=0, \quad X=\beta,
$$

and

$$
\zeta \rightarrow 0, \quad X \rightarrow 0 .
$$

\section{Dispersion relation: concave beach profile}

The general solution (46) satisfies the trapping condition (49) only if $s^{2}>0$ and $B=0$. This leads to the following bound, from (43),

$$
\left(\sigma^{2}-f^{2}\right) /\left(g h_{0}\right)-m^{2}<0,
$$

on frequency and wave number. This bound is simply the high frequency cut-off expected for trapped waves over any monotonic depth profile tending to be a finite depth at infinity $\left(h_{0}\right)$. 
Substitution of (46) with $B=0$ into the coastal boundary condition (48) yields the dispersion relation for edge waves over the margin as

$$
\begin{aligned}
& {\left[s+\frac{m f}{a \sigma}\right] F(s+t, s-t+1,2 s+1 ; \beta)} \\
& \quad+\beta \frac{(s+t)(s-t+1)}{(2 s+1)} F(s+t+1, s-t+2,2 s+2 ; \beta)=0,
\end{aligned}
$$

where the hypergeometric series in (51) are absolutely convergent for $|\beta|<1$ and conditionally convergent for $|\beta|=1$.

In the limit as $\beta \rightarrow 1\left(h_{s} \rightarrow 0\right)$, the coastal boundary conditions is replaced by the requirement (10). Convergence of the solution (46) with $B=0$ then requires that the hypergeometric series terminate, i.e.,

$$
s-t+1=-n, \quad n=0,1,2, \ldots
$$

From (45), (52) may be written as

$$
\frac{m^{2}}{a^{2}}=\frac{m f}{a \sigma}+(n+s)(1+n+s), \quad n=0,1,2, \ldots,
$$

which is the dispersion relation given by [1] for edge waves over the concave exponential profile (39) having zero depth at coastline. For concave exponential profiles having non-zero depth at the coastline $(\beta \neq 1)$ the dispersive properties are defined by (51).

\section{Conclusion}

Exponential depth profiles have been shown to be well suited for approximating the bottom topography of a number of continental margins. The concave exponential has been used successfully in [6] to approximate the near-shore beach environment in a study of high-frequency edge waves. On the other hand the convex exponential profiles have been found useful in studies (e.g. [2]) of low-frequency shelf waves whose trapping scale $0(100 \mathrm{~km})$ requires a model ropography that approximates the broader continental shelf region.

The exact solutions presented in this paper extend the above profiles by providing for an additional adjustable parameter. The presence of three model parameters then allows for independent specification of initial depth at the coastline, shelf slope and shelf width. 


\section{References}

[1] F. K. Ball, "Edge waves in an ocean of finite depth", Deep-Sea Res. 14 (1967), 79-88.

[2] V. T. Buchwald and J. K. Adams, "The propagation of continental shelf waves", Proc. Roy. Soc. A 305 (1968), 235-250.

[3] D. J. Clarke, "High frequency edge waves on an exponential shelf", Dt. Hydrogr. Z. 26 (1973), 265-271.

[4] D. J. Clarke and J. P. Louis, "Edge waves over an exponential continental shelf in a uniformly rotating ocean", Dt. Hydrogr. Z. 28 (1976), 168-179.

[5] A Erdelyi (ed.), Higher transcendental functions. Vol. 1, Bateman manuscript project (McGraw-Hill, New York, 1953).

[6] D A. Huntley and A. J. Bowen, "Field observations of edge waves", Nature 243 (1973), $160-162$.

[7] G Veronis, "Rossby waves with bottom topography", J. Mar. Res. 24 (1966), 338-349. 